\title{
Erratum: Work extraction and fully entangled fraction [Phys. Rev. A 96, 012107 (2017)]
}

\author{
Chung-Yun Hsieh and Ray-Kuang Lee \\ (Received 25 February 2018; published 14 May 2018)
}

DOI: 10.1103/PhysRevA.97.059904

It turns out that the relation between the von Neumann entropy and the fully entangled fraction (FEF) is more complicated than we expected, and Lemma 1 in our paper is valid only under special conditions. As state $\mathcal{J}\left(\mathcal{E}_{\epsilon}^{\dagger}\right)$ in Eq. (B2) is in general un-normalized, the proof of Lemma 1 is incomplete. Even though the inequality given in Lemma 1 can still hold for some states, the absence of Lemma 1 implies that we have to modify our main theorems as Lemma 1 is the core of their proofs. Nevertheless, we derive the modified Theorem 1, which gives a general fully entangled fraction threshold for achieving averaged positive erasure work gain in the identically and independently distributed (iid) limit. This modified result also suggests a thermodynamic interpretation of a fully entangled fraction when one assumes Landauer's principle for a classical observer [1]. Moreover, the modified Theorem 2 gives an upper bound on the difference between $W_{\text {Total }}$ that we introduced in the original paper and the average extractable work in terms of mutual information; in other words, it is a potential tool to estimate the difference of work extractions between two different frameworks.

\section{COUNTEREXAMPLE OF LEMMA 1 IN THE ORIGINAL PAPER}

In general, we have the following inequality for isotropic states: (we define $\mathcal{F}_{+}:=\left\langle\Psi_{d}^{+}\left|\rho_{\text {iso }}\right| \Psi_{d}^{+}\right\rangle$).

Fact 1. Given an isotropic state $\rho_{\text {iso }} \in \mathcal{L}\left(\mathbb{C}^{d} \otimes \mathbb{C}^{d}\right)$, then

$$
S\left(\rho_{\text {iso }}\right) \geqslant-\log _{2} \mathcal{F}\left(\rho_{\text {iso }}\right),
$$

and the equality holds if and only if $\mathcal{F}_{+}=0, \frac{1}{d^{2}}$, or 1 .

Proof. First, we note that the von Neumann entropy of $\rho_{\text {iso }}$ can be written as

$$
S\left(\rho_{\text {iso }}\right)=-\mathcal{F}_{+} \log _{2} \mathcal{F}_{+}-\left(1-\mathcal{F}_{+}\right) \log _{2}\left(\frac{1-\mathcal{F}_{+}}{d^{2}-1}\right) .
$$

Then one can see the equality holds when $\mathcal{F}_{+}=1$. When $\mathcal{F}_{+} \in\left[\frac{1}{d^{2}}, 1\right)$, direct computation shows

$$
\begin{aligned}
S\left(\rho_{\text {iso }}\right)+\log _{2} \mathcal{F}_{+} & =\left(1-\mathcal{F}_{+}\right)\left[\log _{2} \mathcal{F}_{+}-\log _{2}\left(\frac{1-\mathcal{F}_{+}}{d^{2}-1}\right)\right] \\
& =\left(1-\mathcal{F}_{+}\right)\left[\log _{2}\left(\frac{1}{1-\mathcal{F}_{+}}-1\right)+\log _{2}\left(d^{2}-1\right)\right] \\
& \geqslant\left(1-\mathcal{F}_{+}\right)\left[\log _{2}\left(\frac{1}{1-\frac{1}{d^{2}}}-1\right)+\log _{2}\left(d^{2}-1\right)\right]=0 .
\end{aligned}
$$

The equality holds if and only if $\mathcal{F}_{+}=\frac{1}{d^{2}}$. Because $\mathcal{F}\left(\rho_{\text {iso }}\right)=\mathcal{F}_{+}$when $\mathcal{F}_{+} \geqslant \frac{1}{d^{2}}$, this proves the statement for $\mathcal{F}_{+} \in\left[\frac{1}{d^{2}}, 1\right]$. In the region $\mathcal{F}_{+} \in\left[0, \frac{1}{d^{2}}\right)$, we have $\mathcal{F}\left(\rho_{\text {iso }}\right)=\frac{1-\mathcal{F}_{+}}{d^{2}-1}$. Thus, $\mathcal{F}_{+}=0$ implies $\mathcal{F}\left(\rho_{\text {iso }}\right)=\frac{1}{d^{2}-1}$ and $S\left(\rho_{\text {iso }}\right)=\log _{2}\left(d^{2}-1\right)$, which achieves the equality. When $\mathcal{F}_{+} \in\left(0, \frac{1}{d^{2}}\right)$, we have

$$
S\left(\rho_{\text {iso }}\right)+\log _{2} \mathcal{F}\left(\rho_{\text {iso }}\right)=-\mathcal{F}_{+} \log _{2} \frac{\mathcal{F}_{+}}{1-\mathcal{F}_{+}}-\mathcal{F}_{+} \log _{2}\left(d^{2}-1\right) .
$$

Define $f(x):=-x \log _{2} \frac{x}{1-x}-x \log _{2}\left(d^{2}-1\right)$, then we note that on $x \in\left(0, \frac{1}{d^{2}}\right)$ one has

$$
\begin{gathered}
\partial_{x} f(x)=-\log _{2} x+\log _{2}(1-x)-\frac{1}{\ln 2} \frac{1}{1-x}-\log _{2}\left(d^{2}-1\right), \\
\partial_{x}^{2} f(x)=-\frac{1}{\ln 2}\left[\frac{1}{x}+\frac{1}{1-x}+\frac{1}{(1-x)^{2}}\right]<0 .
\end{gathered}
$$

Because $\partial_{x} f(x)>0$ when $x \rightarrow 0$ and since $f(0)=f\left(\frac{1}{d^{2}}\right)=0$, we conclude that $f(x)>0$ on $\left(0, \frac{1}{d^{2}}\right)$. This completes the proof. 
Although this result shows that Lemma 1 in our paper will not hold in general, the inequality of Lemma 1 in our paper still holds for some quantum states. For example, it holds for $\left|\Psi_{d}^{+}\right\rangle$and all the product pure states. Let $\mathcal{D}$ denote the set of quantum states satisfying the inequality of Lemma 1 in our paper, then Theorem 1 in our paper is still true for $\rho \in \mathcal{D}$. This also means Theorem 2 in our paper can still work if we replace condition (i) by $\rho \in \mathcal{D}$. Hence, Fig. 1 still holds but now only for $\rho \in \mathcal{D}$; also, Secs. IV A and IV B (and thus Fig. 2) are true only for $\left|\Psi_{d}^{+}\right\rangle$.

Based on the counterexample illustrated above, our original results become less general than they were thereby being not able to obtain the thermodynamic meaning of the FEF. Nevertheless, with the method to be introduced in the following section, thermodynamic interpretation of the FEF can be obtained; also, although we no longer have $W_{\text {Er }}\left(\rho_{\text {iso }}\right) \approx k_{B} T$ ln $\mathcal{F}\left(\rho_{\text {iso }}\right) d$, the approach presented here gives us an alternative way to conclude a similar result.

\section{Modification of Theorem 1}

In this section, we will prove a result similar to Theorem 1 in our paper.

Modified Theorem 1. Given a quantum state $\rho \in \mathcal{L}\left(\mathbb{C}^{d} \otimes \mathbb{C}^{d}\right)$, then we have

$$
W_{\mathrm{Er}}(\rho) \geqslant g[\mathcal{F}(\rho)] k_{B} T \ln 2,
$$

where $g(x)=\log _{2} d+x \log _{2} x+(1-x) \log _{2}\left(\frac{1-x}{d^{2}-1}\right)$ is strictly increasing on $\left[\frac{1}{d^{2}}, 1\right]$. This means $\rho$ can have work gain under a local erasure process on one party in the iid limit if $\mathcal{F}(\rho)>\mathcal{F}_{\mathrm{Er}}:=g^{-1}(0)$.

Proof. First, for a given quantum state $\rho \in \mathcal{L}\left(\mathbb{C}^{d} \otimes \mathbb{C}^{d}\right)$, there exists a unitary operator $U \in U(d)$ such that

$$
\mathcal{F}(\rho)=\mathcal{F}(\tilde{\rho})=\left\langle\Psi_{d}^{+}|\tilde{\rho}| \Psi_{d}^{+}\right\rangle
$$

where $\tilde{\rho}=(U \otimes \mathbb{I}) \rho(U \otimes \mathbb{I})^{\dagger}$. This means $\mathcal{F}[\mathcal{T}(\tilde{\rho})]=\mathcal{F}_{+}=\mathcal{F}(\tilde{\rho})=\mathcal{F}(\rho)$ where we have $\mathcal{F}_{+}:=\left\langle\Psi_{d}^{+}|\mathcal{T}(\tilde{\rho})| \Psi_{d}^{+}\right\rangle$. Now we note that

$$
-S(A \mid B)_{\mathcal{T}(\tilde{\rho})}=\log _{2} d-S[\mathcal{T}(\tilde{\rho})]=\log _{2} d+\mathcal{F}_{+} \log _{2} \mathcal{F}_{+}+\left(1-\mathcal{F}_{+}\right) \log _{2}\left(\frac{1-\mathcal{F}_{+}}{d^{2}-1}\right)=g\left(\mathcal{F}_{+}\right)=g[\mathcal{F}(\rho)],
$$

where we define $g(x)=\log _{2} d+x \log _{2} x+(1-x) \log _{2}\left(\frac{1-x}{d^{2}-1}\right)$. One can check that $g$ is a strictly increasing function on $x \in$ $\left[\frac{1}{d^{2}}, 1\right]$, which means $g^{-1}$ exists within the interval $\left[-\log _{2} d, \log _{2} d\right]$. Combining with Eq. (4) in our paper, which is obtained from Ref. [1], we obtain

$$
W_{\mathrm{Er}}[\mathcal{T}(\tilde{\rho})] \geqslant g[\mathcal{F}(\rho)] k_{B} T \ln 2 .
$$

We note that, in the approach of Ref. [2], trace preserving completely positive maps that are unital (i.e., preserving the identity operator) can be implemented without work cost, which is also the case in our framework. In this sense, we have $W_{\operatorname{Er}}(\rho) \geqslant W_{\operatorname{Er}}[\mathcal{T}(\tilde{\rho})]$ and $W_{\text {Total }}(\rho) \geqslant W_{\text {Total }}[\mathcal{T}(\tilde{\rho})]$. Hence, we have the following inequality:

$$
W_{\mathrm{Er}}(\rho) \geqslant g[\mathcal{F}(\rho)] k_{B} T \ln 2 .
$$

In particular, it means that $W_{\operatorname{Er}}(\rho)>0$ if $g[\mathcal{F}(\rho)]>0$, which is true if $\mathcal{F}(\rho)>g^{-1}(0)$.

To see the value of $\mathcal{F}_{\mathrm{Er}}:=g^{-1}(0)$, we consider the simplest case when $d=2$. In this two-qubit system, computation shows that $\mathcal{F}_{\mathrm{Er}}^{d=2} \approx 0.8107$, which is much higher than the threshold of usefulness of the standard teleportation 0.5. Asymptotically, this threshold will approach 0.5 when $d \rightarrow \infty$. From here, we also note that, due to this modification, Sec. III A in our paper no longer holds. One can use the $g$ function to calculate results similar to Sec. III B in our paper. More precisely, an isotropic state $\rho_{\text {iso }}$ is local if $W_{\mathrm{Er}}\left(\rho_{\text {iso }}\right) \leqslant g\left(\mathcal{F}_{\mathrm{LHV}}\right) k_{B} T \ln 2[3,4]$; it is unsteerable under general positive operator-valued measures (POVMs) if $W_{\mathrm{Er}}\left(\rho_{\text {iso }}\right) \leqslant g\left[\tilde{p}_{\phi}\left(1-\frac{1}{d^{2}}\right)+\frac{1}{d^{2}}\right] k_{B} T \ln 2$ with $\tilde{p}_{\phi}:=\frac{3 d-1}{d^{2}-1}\left(1-\frac{1}{d}\right)^{d}[4] ;$ it is unsteerable under projective POVMs if $W_{\mathrm{Er}}\left(\rho_{\text {iso }}\right) \leqslant$ $g\left(\frac{H_{d}+H_{d} d-d}{d^{2}}\right) k_{B} T \ln 2[5]$. For a general input state $\rho$, we know that it is not useful for the standard teleportation if $W_{\mathrm{Er}}(\rho) \leqslant$ $g\left(\frac{1}{d}\right) k_{B} T \ln 2[6]$.

\section{Thermodynamic meaning of the fully entangled fraction}

It turns out that the above result suggests a direct thermodynamic interpretation of the fully entangled fraction. By Supplemental Lemma I.5. in Ref. [1], Eq. (10) becomes an equality when we assume Landauer's principle for a classical observer (we refer the reader to the Supplemental Material of Ref. [1] for its definition), which implies the saturation of modified Theorem 1. This means the following result:

Corollary 1. Assume Landauer's principle for a classical observer. For a quantum state $\rho \in \mathcal{L}\left(\mathbb{C}^{d} \otimes \mathbb{C}^{d}\right)$, we have

$$
\mathcal{F}(\rho)=g^{-1}\left[\frac{W_{\operatorname{Er}}(\rho)}{k_{B} T \ln 2}\right] .
$$

In other words, by assuming Landauer's principle for a classical observer, the fully entangled fraction is physically equivalent to the optimal erasure work gain averaged in the iid limit up to a strictly increasing function $g^{-1}$ and an energy scale of $k_{B} T$ ln 2 . 
We further note that in this case the erasure work gain $W_{\mathrm{Er}}$ can be used to witness different nonlocal properties of $\rho$ by using different FEF thresholds [6,7]. For example, given the correctness of Landauer's principle for a classical observer, $\rho$ will be useful for the standard teleportation [6] if $W_{\mathrm{Er}}(\rho)>g\left(\frac{1}{d}\right) k_{B} T \ln 2$; also, there exist fully entangled fraction thresholds $\mathcal{F}_{\mathrm{N}}$ and $\mathcal{F}_{\mathrm{S}}$ [7] such that $\rho$ is nonlocal (steerable) if $\mathcal{F}(\rho)>\mathcal{F}_{\mathrm{N}}\left[\mathcal{F}(\rho)>\mathcal{F}_{\mathrm{N}}\right]$, which implies $\rho$ is nonlocal (steerable) if $W_{\mathrm{Er}}(\rho)>$ $g\left(\mathcal{F}_{\mathrm{N}}\right) k_{B} T \ln 2\left[W_{\mathrm{Er}}(\rho)>g\left(\mathcal{F}_{\mathrm{S}}\right) k_{B} T \ln 2\right]$.

\section{Modification of Theorem 2}

Similar to Theorem 1, we have to modify the proof of Theorem 2 in our paper. In this section, we provide the following result. Note that for a given $\rho \in \mathcal{L}\left(\mathbb{C}^{d} \otimes \mathbb{C}^{d}\right)$, there exists a unitary operator $U \in U(d)$ such that $\tilde{\rho}:=(U \otimes \mathbb{I}) \rho(U \otimes \mathbb{I})^{\dagger}$ with $\mathcal{F}(\rho)=\mathcal{F}(\tilde{\rho})=\left\langle\Psi_{d}^{+}|\tilde{\rho}| \Psi_{d}^{+}\right\rangle$. Here, we also define $\delta_{\epsilon}:=-3 \ln \epsilon$ and see our paper for the definition of $\mathcal{P}_{\rho}^{(1 / 2)-\epsilon}$. then

Modified Theorem 2. Given a quantum state $\rho \in \mathcal{L}\left(\mathbb{C}^{d} \otimes \mathbb{C}^{d}\right)$, if there exists a $\mathcal{P}_{\rho}^{(1 / 2)-\epsilon}$ with $\epsilon \in\left(0, \frac{1}{2}\right]$ that can extract $W_{\text {Total }}(\rho)$,

$$
0 \leqslant W_{\text {Total }}(\rho)-I(A: B)_{\mathcal{T}(\tilde{\rho})} k_{B} T \ln 2<\left\{S[\mathcal{T}(\tilde{\rho})]+\log _{2}\|\rho\|_{\infty}+\delta_{\epsilon}\right\} k_{B} T \ln 2,
$$

where $I(A: B)_{\eta}:=S\left(\eta_{\mathrm{A}}\right)+S\left(\eta_{\mathrm{B}}\right)-S(\eta)$ is the quantum mutual information.

Proof. Recall that trace-preserving completely positive maps that are unital can be implemented with zero work cost in our framework, which implies $W_{\text {Total }}(\rho) \geqslant W_{\text {Total }}[\mathcal{T}(\tilde{\rho})]$. Using Eq. (13) in our paper, we have

$$
W_{\text {Total }}(\rho) \geqslant W_{\text {Total }}[\mathcal{T}(\tilde{\rho})] \geqslant k_{B} T \ln d^{2}-S[\mathcal{T}(\tilde{\rho})] k_{B} T \ln 2=I(A: B)_{\mathcal{T}(\tilde{\rho})} k_{B} T \ln 2 .
$$

If there exists a $\mathcal{P}_{\rho}^{(1 / 2)-\epsilon}$ with $\epsilon \in\left(0, \frac{1}{2}\right]$ which can extract $W_{\text {Total }}(\rho)$, then Ref. [8] implies $\left(\delta_{\epsilon}:=-3 \ln \epsilon\right)$,

$$
W_{\text {Total }}(\rho)<k_{B} T \ln d^{2}-\left[H_{\min }^{\epsilon}(\rho)-\delta_{\epsilon}\right] k_{B} T \ln 2 \leqslant k_{B} T \ln d^{2}+\left(\log _{2}\|\rho\|_{\infty}+\delta_{\epsilon}\right) k_{B} T \ln 2 .
$$

By combining these two inequalities together we obtain

$$
0 \leqslant W_{\text {Total }}(\rho)-I(A: B)_{\mathcal{T}(\tilde{\rho})} k_{B} T \ln 2<\left\{S[\mathcal{T}(\tilde{\rho})]+\log _{2}\|\rho\|_{\infty}+\delta_{\epsilon}\right\} k_{B} T \ln 2 .
$$

The proof is completed.

By using the relation $\left\|\rho_{\text {iso }}\right\|_{\infty}=\mathcal{F}\left(\rho_{\text {iso }}\right)$ for isotropic states $\rho_{\text {iso }}$, we have $0 \leqslant W_{\text {Total }}\left(\rho_{\text {iso }}\right)-I(A: B)_{\rho_{\text {iso }}} k_{B} T \ln 2<\left[S\left(\rho_{\text {iso }}\right)+\right.$ $\left.\log _{2} \mathcal{F}\left(\rho_{\text {iso }}\right)+\delta_{\epsilon}\right] k_{B} T \ln 2$. Due to Fact 1 in this Erratum, we have $S\left(\rho_{\text {iso }}\right)+\log _{2} \mathcal{F}\left(\rho_{\text {iso }}\right) \geqslant 0$, and the equality holds if and only if $\mathcal{F}_{+}=0, \frac{1}{d^{2}}$, or 1 . Hence, this quantity is small only when $\rho_{\text {iso }}$ is very close to the maximally entangled state $\left|\Psi_{d}^{+}\right\rangle$, the maximally mixed state $\frac{\mathbb{I}}{d^{2}}$, or the isotropic state with $\mathcal{F}_{+}=0$. Due to this property, we will no longer have Figs. 1 and 2 in our paper as general schematic interpretations. This also means the conclusions given in Secs. IV A and IV B are too general to be correct due to this modification.

\section{Remark on modified Theorem 2}

We note that both theorems in our paper and modified Theorem 2 have a very strong prerequisite: the existence of a compressionextraction process $\mathcal{P}_{\rho}^{(1 / 2)-\epsilon}$, which can extract $W_{\text {Total }}(\rho)$. However, Eq. (14) is always true without this prerequisite, which helps us to relate $W_{\text {Total }}(\rho)$ to $I(A: B)_{\mathcal{T}(\tilde{\rho})} k_{B} T \ln 2$ where the former is a single shot work extraction averaged in the iid limit and the latter is the average work extraction of the isotropic state $\mathcal{T}(\tilde{\rho})$ (one can see this as the average extractable work by treating the isotropic state as a locally thermal state). Due to this reason, we can write $W_{\max }(\eta):=I(A: B)_{\eta} k_{B} T \ln 2$ for a locally thermal state $\eta$ from now on. We stress that this is an optimal average extractable work, which is by nature quite different from the work extraction of single shot process $W_{\text {Total }}$. Hence, an interesting question is how they are related to each other.

If the above-mentioned prerequisite of modified Theorem 2 is satisfied by a given state $\rho$, then modified Theorem 2 tells us that $W_{\text {Total }}(\rho)$ and $W_{\max }[\mathcal{T}(\rho)]$ are approximately the same up to an error of the energy of $\left(S[\mathcal{T}(\tilde{\rho})]+\log _{2}\|\rho\|_{\infty}+\delta_{\epsilon}\right)$-many qubits. As an example, if the input state is the maximally entangled state $\left|\Psi_{d}^{+}\right\rangle$(or a pure state that is very close to it), we have the approximation to be faithful up to an error of the energy of $\delta_{\epsilon}$-many qubits, which is our old result. This also means $W_{\text {Total }}\left(\left|\Psi_{d}^{+}\right\rangle\right)$and $W_{\max }\left(\left|\Psi_{d}^{+}\right\rangle\right)$can be very similar, even though they are work extractions from two different frameworks. Hence, modified Theorem 2 can be a potential method to justify how close $W_{\text {Total }}$ and $W_{\max }$ are, connecting two different work extraction frameworks together.

\section{Another remark}

Finally, we note that there is a typographical error in Eq. (12): It should be $k \rightarrow \infty$ rather than $k \rightarrow 1$, and Ref. [22] in our paper should be replaced by Ref. [3] in this Erratum. 


\section{ACKNOWLEDGMENT}

We thank Dr. M. Lostaglio for fruitful discussions.

[1] L. del Rio, J. Åberg, R. Renner, O. Dahlsten, and V. Vedral, Nature (London) 474, 61 (2011).

[2] P. Faist, F. Dupuis, J. Oppenheim, and R. Renner, Nat. Commun. 6, 7669 (2015).

[3] N. Brunner, D. Cavalcanti, S. Pironio, V. Scarani, and S. Wehner, Rev. Mod. Phys. 86, 419 (2014).

[4] M. L. Almeida, S. Pironio, J. Barrett, G. Tóth, and A. Acín, Phys. Rev. Lett. 99, 040403 (2007).

[5] H. M. Wiseman, S. J. Jones, and A. C. Doherty, Phys. Rev. Lett. 98, 140402 (2007).

[6] M. Horodecki, P. Horodecki, and R. Horodecki, Phys. Rev. A 60, 1888 (1999).

[7] C.-Y. Hsieh, Y.-C. Liang, and R.-K. Lee, Phys. Rev. A 94, 062120 (2016).

[8] O. C. O. Dahlsten, R. Renner, E. Rieper, and V. Vedral, New J. Phys. 13, 053015 (2011). 